$$
\begin{gathered}
\pi=M \sin \mathrm{x}^{\prime \prime} a ; \varrho=M \sin \mathrm{x}^{\prime \prime} n c \\
p=\pi \operatorname{tg} \alpha ; q=\frac{\pi}{\operatorname{tg} \alpha} ; r=\varrho \sin \delta ; s=\frac{\rho}{\sin \delta}
\end{gathered}
$$

so wird:

$$
\begin{aligned}
& \mathrm{D} \log a=-\frac{M}{a}\left(\frac{\mathrm{d} m}{\mathrm{~d} t}+\frac{\mathrm{d} n}{\mathrm{~d} t} \sin a \operatorname{tg} \delta\right)+r+\frac{\rho n d}{a} \\
& \mathrm{D} \log b=s-p \\
& \mathrm{D} \log c=r-p \\
& \mathrm{D} \log d=q+r \\
& \mathrm{D} \log a^{\prime}=C-p \\
& \mathrm{D} \log b^{\prime}=q \\
& \mathrm{D} \log c^{\prime}=C_{1} \frac{\cos \delta}{c^{\prime}}-C_{2} \frac{d^{\prime}}{c^{\prime}}-\rho \frac{\sin a}{c^{\prime}} \\
& \mathrm{D} \log d^{\prime}=s \cos \delta^{2}-p
\end{aligned}
$$

Die Bestimmung der Aenderungen der Logarithmen der Grössen $b, c, d, a^{\prime}, b^{\prime}, d^{\prime}$ ist also sehr einfach. Nicht so für $a$ und $c$; doch lassen sich auch diese Formeln vielleicht noch bedeutend vereinfachen. So hängt z. B. der erste Ausdruck in $\mathrm{D} \log a$ nur von $a \mathrm{ab}$, und lässt sich schreiben:

$$
\frac{M}{a}\left(\frac{\mathrm{d} m}{\mathrm{~d} t}+\frac{\mathrm{d} n}{\mathrm{~d} t} \cdot \frac{a-m}{n}\right) .
$$

Man kann daher diesen Ausdruck mit dem Argumente $a$ in eine kleine Hülsstafel bringen. Noch einfacher ist es diese hundertjährigen Aenderungen $\mathrm{zu}$ den betreffenden Grössen mit in den Catalog aufzunehmen, wodurch jede Rechnung erspart wird.

Mit den von Herrn Prof. v. Oppolzer gerechneten Constanten wird für die Epoche I 875.0 (Auwers' Fundamental-Catalog für die Zonenbeobachtungen):

$$
\begin{array}{ll}
\varepsilon=23^{\circ} .27^{\prime} 19.93 & \frac{\mathrm{d} \varepsilon}{\mathrm{d} t}=-0.47600 \\
m=46.0664 \mathrm{I} & \frac{\mathrm{d} m}{\mathrm{~d} t}=+0.00028394 \\
n=20.04933 & \frac{\mathrm{d} n}{\mathrm{~d} t}=-0.00008670
\end{array}
$$

Hiermit findet man z. B. für $a$ Cygni:
$\mathrm{D} \log a=+0.00046$
$\mathrm{D} \log b=+0.01323$
$\mathrm{D} \log c=+0.01054$
$\mathrm{D} \log d=-0.00262$
$\mathrm{D} \log a^{\prime}=+0.00770$
$\mathrm{D} \log b^{\prime}=-0.00528$
$\mathrm{D} \log c^{\prime}=-0.0027 \mathrm{r}$
$\mathrm{D} \log d^{\prime}=+0.01057$

während die directe Rechnung:
$\mathrm{D} \log a=+0.0005^{2}$
$\mathrm{D} \log b=+0.01317$
$\mathrm{D} \log c=+0.01047$
$\mathrm{D} \log d=-0.00267$
$\mathrm{D} \log a^{\prime}=+0.0075^{6}$
$\mathrm{D} \log b^{\prime}=-0.00537$
D $\log c^{\prime}=-0.00278$
$\mathrm{D} \log d^{\prime}=+0.01047$

ergab. Für mässige Zeiträume kann man diese Formeln auch noch für dem Pole nicht allzu nahe stehende Sterne verwenden. Für längere Zeiträume werden jedoch für diese sowohl als auch fur Sterne, welche nahe in den Rectascensionen $\alpha=0$ und $\alpha=90^{\circ}$ sind, die Aenderungen nicht mehr durch die ersten Differentialquotienten allein dargestellt werden können. Die höheren Glieder mitzunehmen erweist sich aber als unpraktisch, und wird in diesen Fällen die directe Rechnung vorzuziehen sein.

Wien I 882 Januar.

Norbert Herz.

\title{
Ueber die Bestimmung der Vergrösserung eines Fernrohrs.
}

Im Gesichtsfelde eines Fernrohrs, das mit einem Ocularprisma versehen ist, sieht man ein dunkles Segment. Nennen wir den Abstand des Scheitelpunktes dieses Segments von der Mitte des Gesichtsfeldes $a$, den Brechungswinkel des Prismas $a$, den Brechungsexponenten $n$, den Grenzwinkel $\gamma$, die Vergrösserung $G$, dann wird, vorausgesetzt, dass eine Prismenfläche senkrecht zur optischen Axe des Fernrohrs steht,

$$
\beta=\alpha-\gamma, \sin u=n \sin \beta, G=\frac{\operatorname{tg} u}{\operatorname{tg} a}=\frac{343^{8} \cdot \operatorname{tg} u}{a}
$$

wo $a$ in Bogenminuten ausgedrückt ist.

Wenn wir annehmen, was für Prismen, die man bei den Fernröhren trifft, immer nahe richtig ist, $n=1.53$, $\alpha=45^{\circ}$, so haben wir

$$
G=\frac{386 \cdot 4}{a}
$$

Den Abstand a kann man auf verschiedene Weise bestimmen. So habe ich mit meinem Photometer einen Sterndurchgang beobachtet und $a=23: \mathbf{r}$ gefunden, daraus $G=16.7$. Der genaue Werth der Vergrösserung des Photometers ist $G=\mathrm{r} 6.0$.

Besonders einfach ist die Bestimmung für die Fernrohre, die mit einem Fadennetze versehen sind. Man braucht nur das Prisma dicht am Oculare zu halten und den Abstand $a$ von dem Fadenkreuze in Fadendistanzen zu schätzen.

Wenn das Prisma vorher untersucht ist, wenn man also die strengen Werthe von $n$ und $\alpha$ kennt, so wird man durch genaue Messung von $a$, bei der Beleuchtung des Gesichtsfeldes mit dem monochromatischen Lichte, sehr genau $G$ bestimmen können.

Moskau I 882 Januar I $8 / 30$.

W. Ceraski. 